\title{
Dantzig-Wolfe Decomposition for the Design of Filterless Optical Networks
}

\author{
Brigitte Jaumard, Yan Wang and David Coudert
}

\begin{abstract}
Filterless optical networks use passive splitters and combiners with coherent optics, providing wavelength selection in the digital domain, while forming a passive fiber-tree topology between nodes. In this study, we investigate the optimal design of filterless optical networks while minimizing the number of required wavelengths.

We propose a Dantzig-Wolfe decomposition model in which each subproblem aims to generate a potential filterless optical subnetwork, with a directed tree topology. The master problem then selects the best combination of subnetworks.

Numerical experiments demonstrate significant performance improvement over previous work, reducing previous computational results by a factor of 2 to 10 depending on the size of the data instances.
\end{abstract}

\section{INTRODUCTION}

Optical networks are widely deployed today in all kinds of telecommunication networks, with a volume of user traffic on carrier networks growing at a rate between $20 \%$ and $40 \%$ per year during the last decade [1]. As exponential traffic growth was set to slow, the rise of $5 \mathrm{G}$ introduced new use cases, with capacity growth set to continue. In this context, from a techno-economic point of view, filterless optical networks offer opportunities for cost and energy reduction, at least for certain types of networks, for example access networks [2].

There is a compromise to be found between the usage of more spectrum efficient technologies thanks to, e.g., pure optical layer switching using expensive Reconfigurable Optical Add-Drop Multiplexers (ROADMs) (see, e.g., Simons [3]), and filterless optical networks which rely on cheap couplers/splitters at the expense of a reduction in the spectral efficiency performance [4].

Tzanakaki et al. [5] was one of the pioneers to investigate the filterless network components. The basic design principle of filterless optical networks was described by Savoie et al. [6] in the early work on filterless optical networks with a motivation for cost reduction and environment friendly networks. In spite of a decrease in the spectrum efficiency, more authors are studying filterless optical networks today,

B. Jaumard has been supported by a Concordia University Research Chair (Tier I) on the Optimization of Communication Networks and by an NSERC (Natural Sciences and Engineering Research Council of Canada) grant. D. Coudert has been supported by the French National Research Agency (ANR), through the UCA ${ }^{\mathrm{JEDI}}$ Investments in the Future project with the reference number ANR-15-IDEX-0001, and the Inria associated-team project EfDyNet (Efficient Dynamic Resource Allocation in Networks).

B. Jaumard is with the Department of Computer Science and Software Engineering, Concordia University, Montreal, QC, Canada.

Y. Wang is with the Department of Computer Science and Software Engineering, Concordia University, Montreal, QC, Canada.

D. Coudert is with Université Côte d'Azur, Inria, CNRS, I3S, Sophia Antipolis, France. and we can see studies investigating the wasted spectrum on the static planning case (filterless) vs. dynamic planning case (filtered node), see, e.g., Pavon-Marino et al. [2]. The addition of blockers offers a mean to improve the efficiency of spectrum utilization, see, e.g., Dochhan et al. [7].

Several researchers study the design of optimized filterless optical networks. Tremblay et al. [8], Ayoub et al. [9] and Jaumard et al. [10] all proposed a two-step solution process with, for the first two references, a first step aiming at generating potential/promising fiber trees, and then a second step to provision the resulting trees. The quality of the resulting filterless optical networks is however difficult to assess, both for the two-step process, but also because one or both steps are solved using heuristics. For instance, Tremblay et al. [8] use heuristics for both steps: a genetic algorithm for the fiber link interconnexion (definition of filterless trees and subnetworks), and a Tabu Search meta-heuristic for the wavelength assignment.

In [11], there is a first attempt for designing an exact method for the optimal design of filterless optical networks, using a decomposition mathematical program. However, computational times are in the order of hours. In this paper, we improve on that latter study, with an improved mathematical programming model, as well as an improved exact solution process. This translates into significant reduced computational times and further improved accuracy for the output designs.

More recently, other studies have addressed additional features of filterless networks, e.g., survivability [12], bidirectional single-fiber filterless optical networks [13], programmable filterless networks [14].

The paper is organized as follows. In Section II, we present the problem statement and the concept of filterless optical network design. In Section III, we propose a new mathematical model DW_FOP for the optimal design of filterless optical networks. The solution process, which relies on Dantzig-Wolfe decomposition (or column generation techniques), is described In Section IV. Computational results are summarized in Section $\mathrm{V}$, including a comparison with the results of previous works. Conclusions are drawn in the last section.

\section{Design of Filterless Networks}

Consider an optical network represented by its physical network $G=(V, L)$, where $V$ is the set of nodes (indexed by $v$ ), and $L$ is the set of fiber links (indexed by $\ell$ ). We denote the set of incoming and outgoing links of $v$ as $\operatorname{IN}(v)$ and $\operatorname{OUT}(v)$, respectively.

The traffic is described by a set of unit requests $K$, where each request $k$ is characterized by its source and destination 


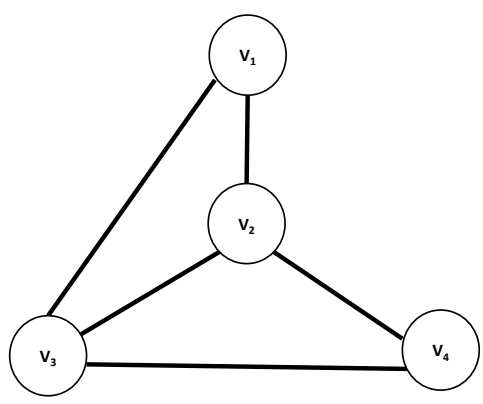

(a) Physical network

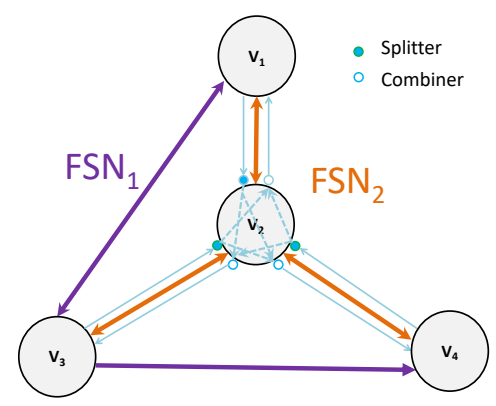

(b) Two FSNs

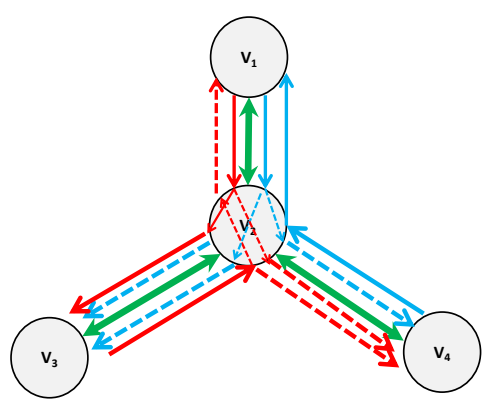

(c) Wavelength provisioning of $\mathrm{FSN}_{2}$

Fig. 1: Construction of a filterless network solution

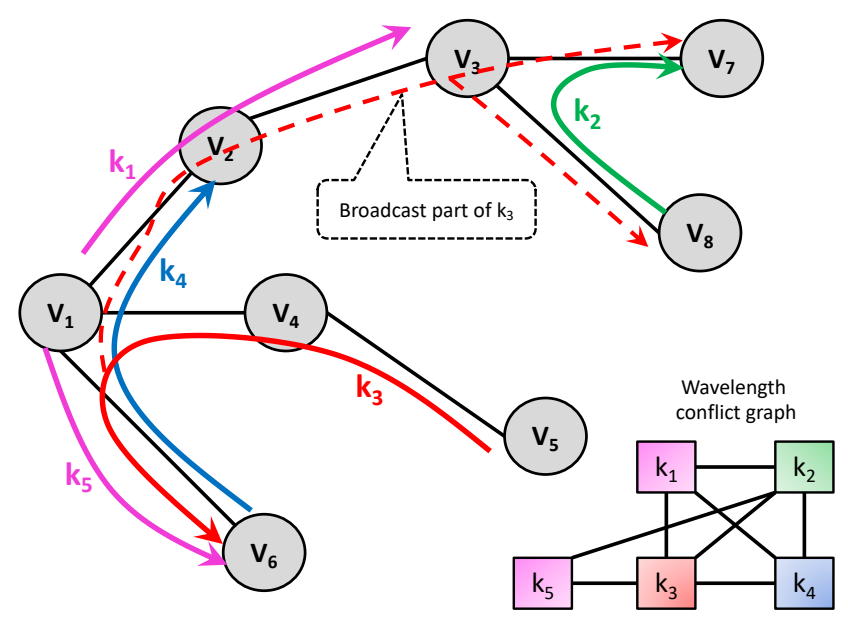

Fig. 2: Requests with wavelength conflicts

nodes, $v_{s}$ and $v_{d}$ respectively. Indeed, non unit requests are considered as a set of unit requests.

The architecture provides at least one optical path between each node pair [2] and is expected to offer the following significant advantages [3]: 1 . Eliminating or reducing the usage of active photonic switching elements. 2. Reducing initial deployment costs. 3. Ease of maintenance and reconfigurability, good resilience, and multicast capability.

Filterless networks corresponds to passive networks in which passive optical splitters and combiners are used for interconnecting fiber links. Their advantages lie in: (i) the elimination/reduction of the usage of active photonic switching elements, (ii) the reduction of the initial deployment costs and (iii) the ease of of maintenance and reconfigurability, good resilience, and multicast capability.

Due to signal broadcast and the absence of filtering, some of the signals appear on unintended links which can lead to an overhead in spectrum usage and an increase of the blocking probability. Indeed, although blockers can be used, the unfiltered channels propagate after reaching their destination, the signal from each transmitter is broadcasted to all branches of the corresponding fiber tree (see definition below) downstream of the source node.
A filterless network solution consists of a set of filterless optical sub-network (FSN for short) solutions, where each FSN relies on an undirected tree, called the backbone of an FSN, deduced from a subgraph of the physical network $G$. FSNs need to be pairwise fiber link disjoint in order to avoid closed loops, i.e., undesirable laser effects in optically amplified links [8].

Wavelength assignment follows the usual rule of avoiding wavelength conflicts, see the discussion below. On the undirected tree, we use the term "edge" to represent an undirected link, which can host multiple directed links or links for short, possibly in both directions. We denote the set of adjacent links of $v$ as $\omega(v)$.

Figure 1 illustrates the construction of a filterless optical network made of two FSNs: the red one $\left(\mathrm{FSN}_{1}\right)$ is supported by the undirected tree $T_{1}=\left\{\left\{v_{1}, v_{3}\right\},\left\{v_{3}, v_{4}\right\}\right\}$, and the green one $\left(\mathrm{FSN}_{2}\right)$, by the undirected tree $T_{2}=$ $\left\{\left\{v_{1}, v_{2}\right\},\left\{v_{2}, v_{3}\right\},\left\{v_{2}, v_{4}\right\}\right\}$.

In Figure 1(b), fiber links are represented by directed arrows, where a bidirected arrow indicates two fiber links, one in each direction. The first FSN, i.e., $\mathrm{FSN}_{1}$, is made of one bi-directional arrow, and a single fiber link on the second tree edge supporting the FSN. The second FSN, i.e., $\mathrm{FSN}_{2}$ has two opposite fiber links on each edge of its supporting undirected tree.

On the FSN intermediate nodes, passive splitters and combiners are required to interconnect the fiber links supporting the request provisioning, see, e.g., node $v_{2}$ requires three splitters/combiners with two ports in order to accommodate the provisioning of the requests using the green filterless network, see Figure 1(b).

On our example, each request can be provisioned on any one of the sub-networks. While this is not necessarily the case for all requests in general, this may happen in practice for some requests. In the example of Figure 1(b), the request from node $v_{1}$ to node $v_{4}$ can be provisioned either on $\mathrm{FSN}_{1}$ or $\mathrm{FSN}_{2}$. The decision is usually driven by the objective, e.g., number of wavelengths, number of fiber trees, number of passive optical splitters. In this study, we are interested in establishing a set of FSNs such that the provisioning of all the requests minimizes the number of wavelengths.

Figure 1(c) depicts a possible wavelength assignment, with 
the provisioning of 4 requests on $\mathrm{FSN}_{2}$ in Figure 1(b): $k_{1 \mapsto 3}$ from $v_{1}$ to $v_{3}$ with the red wavelength (plain lines) and its broadcast effect (dashed lines), and with the same notations, $k_{1 \mapsto 2}$ with the blue wavelegnth, $k_{4 \mapsto 1}$ with the blue wavelength, and $k_{3 \mapsto 2}$ with the red wavelength. Note that in the broadcast part, we can allow wavelength re-use, e.g., the red wavelength on link $\left(v_{2}, v_{4}\right)$ (a first broadcast effect from $k_{1 \mapsto 2}$, and a second one from $k_{3 \mapsto 2}$ ).

We now consider the broadcast effect. When the destination is reached, the lightpath will continue downstream. For instance, when the optical signal supporting request $k_{1 \mapsto 2}$ reaches $v_{2}$ through the fiber link $\left(v_{1}, v_{2}\right)$, the signal is broadcast through fiber links $\left(v_{2}, v_{3}\right)$ and $\left(v_{2}, v_{4}\right)$.

Wavelength assignment should be done in order to avoid wavelength conflicts. Such conflicts occur for two requests if there is a shared fiber link on one of their routing path, i.e., between source and destination nodes. However, requests that share links for broadcasting beyond the destination, are not considered conflicting and may share the same wavelength. For instance, in Figure 2, there are conflicts for the requests $k_{1}$ and $k_{4}$ because their routes between the source and destination nodes share a fiber link. Requests $k_{1}$ and $k_{2}$ are also wavelength conflicting as one of the fiber links of $k_{2}$ lies on the broadcast route of $k_{1}$ with unfiltered channels. Similarly, requests $k_{3}$ and $k_{4}$ are wavelength conflicting, as $k_{4}$ is on the broadcast of $k_{3}$. Non conflicting requests are for instance $k_{1}$ and $k_{5}$, or $k_{4}$ and $k_{5}$. Note that once an FSN is defined, wavelength assignment can be done with a graph coloring algorithm (see, e.g., [8]), using a wavelength graph conflict, as illustrated in Figure 2.

In Figure 1(c), requests $k_{1 \mapsto 3}$ and $k_{3 \mapsto 2}$ are not conflicting and so can be assigned the same wavelength. The same holds between requests $k_{1 \mapsto 2}$ and $k_{4 \mapsto 1}$. For this example, 2 wavelengths are sufficient to satisfy the 4 requests.

\section{MATHEMATICAL Model}

\section{A. Definitions and Notations}

We introduce the concept of FSN configuration as a provisioned directed tree characterized by:

- $a_{\ell}^{\mathrm{F}}=1$ if link $\ell$ is in configuration $\mathrm{F}, 0$ otherwise.

- $x_{k}^{\mathrm{F}}=1$ if request $k$ is routed on configuration $\mathrm{F}, 0$ otherwise. Indeed, if it happens that a request is provisioned in two different FSNs due to constraints (3), then one of them is arbitrarily chosen (using a post-processing).

Note that the reason of the inequalities in constraints (3) is motivated by the greater easiness of solving the mathematical program with inequalities, than with equalities. Expressing constraints (3) with equality would eliminate this issue, at the expense of longer computational times.

- $\beta_{\lambda}^{\mathrm{F}}=1$ if wavelength $\lambda$ is used in configuration $\mathrm{F}, 0$ otherwise.

Denote by $\mathcal{F}$ the overall set of FSN configurations, and let $n_{\mathcal{F}}$ be an upper bound on the number of FSNs in the network.

\section{B. Mathematical Model}

We propose an integer linear program that has two sets of decision variables:
- $z^{\mathrm{F}}=1$ if FSN configuration is selected as a filterless subnetwork, 0 otherwise. Note that the conflicts between the requests routed on $\mathrm{F}$ have been taken into account in the definition of $\mathrm{F}$ and the wavelength assignment is encoded in F.

- $x_{\lambda}=1$ if wavelength $\lambda$ is used by any of the selected FSN configurations, 0 otherwise.

The objective corresponds to the minimization of the total number of wavelengths.

$$
\min \sum_{\lambda \in \Lambda} x_{\lambda}
$$

subject to:

$$
\begin{array}{ll}
\sum_{\mathrm{F} \in \mathcal{F}} \beta_{\lambda}^{\mathrm{F}} z^{\mathrm{F}} \leq n_{\mathcal{F}} x_{\lambda} & \lambda \in \Lambda \\
\sum_{\mathrm{F} \in \mathcal{F}} x_{k}^{\mathrm{F}} z^{\mathrm{F}} \geq 1 & \\
\sum_{\mathrm{F} \in \mathcal{F}} a_{\ell}^{\mathrm{F}} z^{\mathrm{F}} \leq 1 & \\
\sum_{\mathrm{F} \in \mathcal{F}} z^{\mathrm{F}} \leq n_{\mathcal{F}} & \\
z^{\mathrm{F}} \in\{0,1\} & \mathrm{F} \in \mathcal{F} \\
x_{\lambda} \in\{0,1\} & \lambda \in \Lambda .
\end{array}
$$

Constraints (2) indicate that a wavelength is used in the solution if it is used by any of the selected FSN. Since the FSNs are link disjoint, the same wavelength $\lambda$ can be used by several FSNs. The constraint is presented as a "big-M" constraint, that is a constraint in which the right-hand side is a large enough constant (i.e., that is always larger than the value of the left-hand side) times a binary variable. Here, the value $n_{\mathcal{F}}$ is sufficient since Constraint (5) limits the number of FSNs to be selected to $n_{\mathcal{F}}$. Constraints (3) ensure that each request is routed on at least one selected FSN. Observe that, although a request should be routed on "a unique" FSN, it is faster in practice for LP/ILP solvers to find a solution when the constraint is relax to "at least one" FSN, and then to use a simple post-processing procedure to extract a unique route per request from the solution. Constraints (4) ensure that selected FSNs are link disjoint (i.e., that a link can be used by at most one selected FSN). Constraint (5) limits the number of FSNs to be selected. Finally, Constraints (6) and (7) define the domains of the variables.

As can be observed, the above model (1) - (7) has an exponential number of variables, and corresponds to a DantzigWolfe decomposition model, which can be solved by a column generation algorithm, and, e.g., another algorithm to derive an ILP solution, without the requirement of explicitly enumerating all the variables.

\section{Solution Process}

We propose to use a solution process, in which the column generation technique is used to solve the linear relaxation of problem (1)-(7). Section III presented the mathematical model of the so-called master problem in the context of 
Dantzig-Wolfe decomposition associated with column generation algorithm. We proposed two pricing models to compute the new improved FSN configuration. In section IV-A, we propose a one step full pricing model, which includes routing and wavelength assignment inside the same mathematical formulation. In Section IV-B, we present a simplified version of pricing model, which leads to a routing only configuration as a first step. Then, we apply graph coloring on the obtained configuration to assign wavelengths to requests, and finally we adjust the reduce cost calculation accordingly. In Section IV-C, we provide a detailed description on how to coordinate these two models.

\section{A. FSN Pricing Problem}

This pricing problem aims to find new "improving" FSN configurations, i.e., a configuration such that if added to the current Restricted Master Problem (RMP) will improve the value of its linear relaxation, where an FSN configuration is defined by the FSN backbone (undirected tree supporting the FSN), its set of links supporting the routing of the requests, and the wavelength assignment.

Variables. Note that several variables of the pricing problem correspond to parameters of the Master Problem. In order to alleviate the notations, and with a slight abuse of notations, we denote them the same way.

- $a_{\ell}=1$ if link $\ell$ is in the FSN under construction, 0 otherwise, for all $\ell \in L$

- $a_{v}=1$ if node $v$ belongs to the FSN under construction, 0 otherwise, for all $v \in V$

- $\alpha_{e}=1$ if edge $e$ belongs to the backbone of the selected tree under construction, 0 otherwise, for all $e \in E$.

- $x_{k}=1$ if request $k$ is routed on the FSN under construction, 0 otherwise, for all $k \in K$

- $\varphi_{k \ell}=1$ if the routing of request $k$ goes through link $\ell$, or if the channel used in the routing of request $k$ propagates on link $\ell$ because it is not filtered, 0 otherwise, for all $\ell \in$ $L, k \in K$

- $\psi_{k \ell}=1$ if the routing of request $k$ goes through link $\ell$ between its source and destination, 0 otherwise, for all $\ell \in$ $L, k \in K$

- $\theta_{k k^{\prime}}=1$ if request $k$ and request $k^{\prime}$ are in conflict on the FSN under construction, 0 otherwise.

- $w_{k k^{\prime} \ell}=\psi_{k \ell} \varphi_{k^{\prime} \ell}$, for all $\ell \in L, k, k^{\prime} \in K$. In other words, with $w_{k k^{\prime} \ell}=1$ identifying a link on which $k$ and $k^{\prime}$ are conflicting either between their source and destination nodes, or with one of them being routed to the broadcast of the other request, and 0 otherwise (no conflict).

- $\beta_{\lambda}=1$ if the wavelength $\lambda$ is used on the FSN under construction, 0 otherwise, for all $\lambda \in \Lambda$

- $\beta_{\lambda k}=1$ if request $k$ is assigned the wavelength $\lambda$ on the FSN under construction, 0 otherwise, for all $k \in K, \lambda \in \Lambda$

The objective of the pricing problem with respect to the $z^{\mathrm{F}}$ variables (or columns made up of their coefficients in the constraint matrix of the master problem) corresponds to the reduced cost of these variables, following the Dantzig-Wolfe decomposition algorithm which equals the simplex algorithm with an implicit enumeration of variables. Therefore, variables of the pricing problem are weighted with the values of dual variables of the master problem, and we refer the reader who is not familiar with (delayed) linear programming (aka DantzigWolfe algorithm) to the seminal book of Chvátal [15]. Objective:

$$
\min 0+\sum_{\lambda \in \Lambda} u_{\lambda}^{(2)} \beta_{\lambda}-\sum_{k \in K} u_{k}^{(3)} x^{k}+\sum_{\ell \in L} u_{\ell}^{(4)} a_{\ell}+u^{(5)}
$$

subject to:

\section{Construct an undirected tree}

$$
\begin{array}{ll}
\sum_{\substack{e=\left\{v, v^{\prime}\right\} \in E: \\
v, v^{\prime} \in V^{\prime}}} \alpha_{e} \leq\left|V^{\prime}\right|-1 & V^{\prime} \subseteq V,\left|V^{\prime}\right| \geq 3 \\
\sum_{v \in V} a_{v}=\sum_{e \in E} \alpha_{e}+1 & \\
2 \alpha_{e} \leq a_{v}+a_{v^{\prime}} & v, v^{\prime} \in V, e=\left\{v, v^{\prime}\right\} \\
\sum_{e \in \omega(v)} \alpha_{e} \geq a_{v} & v \in V \\
a_{\ell} \leq \alpha_{e}, a_{\bar{\ell}} \leq \alpha_{e} & \ell=\left(v, v^{\prime}\right), \bar{\ell}=\left(v^{\prime}, v\right) \\
& v, v^{\prime} \in V, e=\left\{v, v^{\prime}\right\}
\end{array}
$$

\section{Routing of the requests}

$$
\begin{array}{ll}
\varphi_{k \ell} \leq a_{\ell} & k \in K, \ell \in L \\
a_{\ell} \leq \sum_{k \in K} \varphi_{k \ell} & \ell \in L \\
\varphi_{k \ell}+\varphi_{k \bar{\ell}} \leq 1 & \ell=\left(v, v^{\prime}\right), \bar{\ell}=\left(v^{\prime}, v\right): \\
& v \in V, v^{\prime} \in V
\end{array}
$$

Flow constraints for broadcast stream

$$
\begin{array}{cc}
\sum_{\ell \in \mathrm{IN}\left(d_{k}\right)} \varphi_{k \ell}=\sum_{\ell \in \operatorname{OUT}\left(s_{k}\right)} \varphi_{k \ell}=x_{k} & k \in K \\
\sum_{\ell \in \mathrm{IN}\left(s_{k}\right)} \varphi_{k \ell}=0 & k \in K \\
\varphi_{k \ell^{\prime}} \leq \sum_{\ell \in \mathrm{IN}(v)} \varphi_{k \ell}+1-a_{\ell^{\prime}} & k \in K, \\
\varphi_{k \ell} \leq \varphi_{k \ell^{\prime}}+2-a_{\ell}-a_{\ell^{\prime}} & v \in V \backslash\left\{s_{k}\right\}, \ell^{\prime} \in \operatorname{OUT}(v) \\
v \in V, \ell \in \mathrm{IN}(v), \ell^{\prime} \in \operatorname{OUT}(v) \backslash\{\bar{\ell}\}
\end{array}
$$

\section{Flow constraints between source and destination}

$$
\begin{array}{ll}
\sum_{\ell \in \mathrm{IN}\left(d_{k}\right)} \psi_{k \ell}=\sum_{\ell \in \operatorname{OUT}\left(s_{k}\right)} \psi_{k \ell}=x_{k} & k \in K \\
\sum_{\ell \in \mathrm{IN}(v)} \psi_{k \ell}=\sum_{\ell \in \operatorname{OUT}(v)} \psi_{k \ell} \leq x_{k} & k \in K, \\
\sum_{\ell \in \mathrm{OUT}\left(d_{k}\right)} \psi_{k \ell}=\sum_{\ell \in \mathrm{IN}\left(s_{k}\right)} \psi_{k \ell}=0 & k \in K \backslash\left(s_{k}, d_{k}\right) \\
\psi_{k \ell} \leq \varphi_{k \ell} & k \in K, \ell \in L \\
\sum_{\ell \in L} \operatorname{DIST}_{\ell} \psi_{k \ell} \leq \text { REACH_DIST } & k \in K
\end{array}
$$




\section{Identify wavelength conflicting paths}

$$
\begin{array}{ll}
\theta_{k k^{\prime}} \geq \psi_{k \ell}+\psi_{k^{\prime} \ell}-1 & \ell \in L, k, k^{\prime} \in K \\
\theta_{k k^{\prime}} \geq \psi_{k \ell}+\varphi_{k^{\prime} \ell}-1 & \ell \in L, k, k^{\prime} \in K \\
\theta_{k k^{\prime}} \geq \psi_{k^{\prime} \ell}+\varphi_{k \ell}-1 & \ell \in L, k, k^{\prime} \in K \\
\theta_{k k^{\prime}} \leq \sum_{\ell \in L}\left(w_{k k^{\prime} \ell}+w_{k^{\prime} k \ell}\right) & k, k^{\prime} \in K \\
w_{k k^{\prime} \ell} \leq \psi_{k \ell} & \\
w_{k k^{\prime} \ell} \leq \varphi_{k^{\prime} \ell} & \ell \in L, k, k^{\prime} \in K \\
w_{k k^{\prime} \ell} \geq \psi_{k \ell}+\varphi_{k^{\prime} \ell}-1 & \ell \in L, k, k^{\prime} \in K \\
&
\end{array}
$$

\section{Wavelength Assignment}

$$
\begin{aligned}
& \sum_{\lambda \in \Lambda} \beta_{\lambda k}=x_{k} \\
& \beta_{\lambda k} \leq \beta_{\lambda} \\
& \beta_{\lambda k}+\beta_{\lambda k^{\prime}} \leq 2-\theta_{k k^{\prime}}
\end{aligned}
$$$$
k \in K
$$

$$
\begin{aligned}
& \lambda \in \Lambda, k \in K \\
& \lambda \in \Lambda, k, k^{\prime} \in K
\end{aligned}
$$

\section{Definition of variables}

$$
\begin{array}{ll}
\alpha_{e} \in\{0,1\} & e=\left\{v, v^{\prime}\right\} \in E \\
a_{v} \in\{0,1\} & v \in V \\
a_{\ell} \in\{0,1\} & \ell \in L \\
\varphi_{k \ell} \in\{0,1\} & \ell \in L, k \in K \\
\psi_{k \ell} \in\{0,1\} & \ell \in L, k \in K \\
x_{k} \in\{0,1\} & k \in K \\
\theta_{k k^{\prime}} \in\{0,1\} & k, k^{\prime} \in K \\
\beta_{\lambda} \in\{0,1\} & \lambda \in \Lambda \\
\beta_{\lambda k} \in\{0,1\} & \lambda \in \Lambda, k \in K . \\
w_{k k^{\prime} \ell} \in\{0,1\} & k, k^{\prime} \in K, \ell \in L .
\end{array}
$$

Constraints (9) - (13) aim to restrict the undirected tree condition. Constraints (9) are the classical subtour elimination constraints in order to guarantee an acyclic graph structure (i.e., a supporting tree for the f-subnet under construction) [16].

As the number of these constraints is exponential, we will enumerate them only implicitly throughout a so-called lazy constraint procedure [17]. That is, we first solve the model with (9) restricted to a single constraint, e.g., $V^{\prime}=V$ only. Then, if the set of selected edges is not connected (and so may contain a cycle), we add a constraint (9) for each subset of vertices $V^{\prime}$ corresponding to the vertices of a connected component of the set of selected edges and solve again the model. We repeat this process until all constraints are satisfied. In practice, the number of introduced constraints is much smaller than the exponential number of constraints associated with (9).

Constraint (10) ensures that the selected edges correspond to the edges of a tree and so that a unique f-subnet will be generated in the entire network. Constraints (11) and (12) guarantee the consistency between node and edge variables: an edge is used in the undirected tree if and only if its two endpoints belong to it. Constraints (13) enforce the consistency between link and edge variables: if a link is used, its associated edge belongs to the undirected tree. Here, the notation $\bar{\ell}$ denotes the link in the opposite direction of link $\ell$.

Constraints (14) - (16) take care of the routing of the requests, including the links hosting the unfiltered channels. Constraints (14) and (15) ensure that a request can be routed over link $\ell$ if and only if that link $\ell$ belongs to the f-subnet under construction and vice versa, if a link belongs to the output f-subnet structure, then at least one routing uses it. Constraints (16) prevent the use of both $\ell$ and $\bar{\ell}$ in the routing of a given request, and so ensure that the signal can not be sent back to the sender.

Constraints (17) - (20) are the flow constraints for the requests, but include the broadcast effect. Constraints (17) states for each request, the total incoming traffic which associated to this request on the destination node should be 1 if this request is routed on this f-subnet, 0 otherwise, so as the total outgoing traffic for the source node of the request. Constraints (18) ensure that no flow supporting a request $k$ can enter its source node $s_{k}$.

The next two sets of Constraints (19) - (20) are for taking care of the propagation of the unfiltered channels. Constraints (19) enforce for every request $k$ and node different from its source node $\left(s_{k}\right)$, that if none of its incoming link is on the route or broadcast effect of request $k$, then none of the outgoing links can be used for either the routing or the broadcast effect of request $k$. Constraints (20) make sure on each node, for every request $k$, that any used outgoing links will carry the traffic for this request if there is incoming traffic of this request. This is to enforce that the broadcast effect will carry over to the traffic going forward. In other words, if $\varphi_{k \ell}=a_{\ell}=1$, it will force $\varphi_{k \ell^{\prime}}=1$ if $a_{\ell^{\prime}}=1$, i.e., if link $\ell^{\prime}$ belongs to the FSN under construction.

Constraints (21) - (22) are flow constraints that define the routing of request $k$ between its source and destination. Constraints (23) impose no wavelength assignment on the incoming links of the source and on the outgoing links of the destination for the "filtered" routing of $k$.

Constraints (25) are the reach constraints. That is, for each request the routing distance between source and destination must not exceed a maximum distance $(1,500 \mathrm{~km})$. Constraints (24) define the relation between variables $\varphi_{k \ell}$ and $\psi_{k \ell}$, which indicate $\psi_{k \ell}$ is the subset of $\varphi_{k \ell}$.

Constraints (26) - (28) are wavelength conflicts constraints expressing that: either $k$ and $k^{\prime}$ share a link between their source and destination nodes, see (26), or that one of the requests is routed to the broadcast part of the other request, see (27) and (28). Constraints (29) - (32) are the linearization of the constraints defining variables $w_{k k^{\prime} \ell}$, that is $w_{k k^{\prime} \ell}=$ $\psi_{k \ell} \varphi_{k^{\prime} \ell}$.

Constraints (33) express that one and only one wavelength is used per granted request, and that no wavelength is used if the request is not granted. Constraints (34) indicate that a wavelength is used in the FSN configuration if any of the granted request uses it. Constraints (35) enforce conflicting requests to use different wavelengths. 


\section{B. Relaxed FSN Pricing Problem}

The pricing problem described in Section IV-A is a quite complex optimization problem. In order to speed up the solution process, we used a two-step process in the first iterations of column generation to solve the pricing problem.

The two step solution process consists in solving first a relaxation of the FSN pricing problem defined as the FSN pricing problem in which we omit the constraints related to the wavelength assignment, i.e., Constraints (26) to (35). We can then simplify the objective function of the pricing problem, and omit the term $\sum_{\lambda \in \Lambda} u_{\lambda}^{(2)} \beta_{\lambda}$.

This relaxation of the pricing problem outputs an FSN backbone, jointly with a routing for the granted requests. Once the relaxed FSN pricing problem is solved, we perform the wavelength assignment, which we reformulate as a graph coloring problem on the wavelength conflict graph. We then use the graph coloring algorithm of [18] and recompute the reduced cost in order to determine if we end up with an improving FSN configuration.

\section{Detailed Solution Process}

Figure 3(a) presents the detailed process of the two step solution of the FSN pricing problem. Figure 3(b) shows the overall solution process, using first the two step solution process of the FSN pricing problem, and then the one step process. The advantage of two step pricing model is to reduce the computational burden, especially when we solve data instances with larger networks. After the two step pricing model satisfies the optimality condition, the FSN pricing problem is solved at least once, in order to guarantee the optimal solution.

Solution process steps:

1) Generate an initial solution:

a) Compute an FSN supported by a spanning tree, which grants all the requests

b) Compute the wavelength assignment on the FSN generated by step 1a (with, e.g., the ILP model of Mehrotra et al. [18]).

2) Apply the column generation algorithm in order to solve the linear relaxation of the model of DW_FOP:

a) Solve restricted master problem with current FSNs

b) Solve the two step relaxed FSN pricing problem:

i) Compute an FSN with the relaxed FSN pricing problem (see definition in Section IV-B)

ii) Calculate the wavelength assignment on this FSN

iii) Re-compute the reduced cost on the resulted provisioned FSN

iv) If it generates an improving FSN configuration, add it to the RMP and return to Step 2a

c) Solve one step full pricing problem with the mathematical model of IV-A. If it generates an improving wavelength configuration, add it to the RMP and return to Step $2 \mathrm{a}$

3) Continuous relaxation of the Master Problem has been solved optimally

4) Solve the last generated restricted master problem with integer requirements for the variables, derive an ILP solution.
Note that in the particular case of a single FSN, convergence is very fast, and therefore, we do not need to recourse to an initial solution to speed up the solution process. As we can see in Section V, in practice, either we directly generate an optimal spanning tree, or we find it after a first attempt. When searching for more than one FSN, recourse to an initial solution is needed in order to improve the convergence speed, and reduce the computational times. However, it is enough to generate an initial solution with a unique spanning tree.

\section{Numerical Results}

In this section, we present our computational results, which we compare both to those of the literature [8] and to our own previous results [11].

The software used to solve LP and ILP problems is the IBM ILOG CPLEX Optimization Studio version 12.8, and we use $\mathrm{C}++$ to interact with its API. We run the programs on a x64 based processor, Intel(R) CPU E3-1270 v5 @ 3.6 GHz; RAM $32 \mathrm{~GB}$, with a Windows 10 operating system.

\section{A. Data Sets}

We used the same network topologies as Tremblay et al. [8], i.e., Italy, California, and Germany. We added two networks, also widely used in other papers on optical networking: Cost239 and USA, from [19]. We recall their characteristics in Table I, i.e., the number of nodes and edges of the network topologies, as well as their number of requests. We consider a first set of requests, with a one unit wavelength requirement for each pair of nodes, as in [8]. We then added a second set of uniformly randomly generated requests on the pairs of nodes, with a random number of units in $\{0,1,2,3,4\}$.

\begin{tabular}{|l|c|c|c|c|}
\hline \multirow{2}{*}{ Networks } & \multirow{2}{*}{ \# nodes } & \multirow{2}{*}{ \# edges } & \multicolumn{2}{|c|}{ \# requests } \\
\cline { 4 - 5 } & & & Set \#1 & Set \#2 \\
\hline Italy & 10 & 15 & 90 & 180 \\
California & 17 & 20 & 272 & 544 \\
Germany & 17 & 26 & 272 & 544 \\
Cost239 & 11 & 26 & 110 & 220 \\
USA & 12 & 15 & 132 & 264 \\
\hline
\end{tabular}

TABLE I: Characteristics of network topologies and requests

\section{B. Comparative computational results}

We summarize our computational results on different number of FSNs in Tables II, III, and IV.

Each table contains the following results:

- $z_{\mathrm{LP}}$ : the linear programming value

- Model CG_FOP: as we limited the number of solutions of the wavelength pricing problem to 500, the resulting LP value is the last computed one, and not necessarily an optimal one. It is denoted by $\tilde{z}_{\mathrm{LP}}$ and is not a lower bound.

- Model DW_FOP: it is the optimal one, and is denoted by $z_{\mathrm{LP}}^{\star}$.

-\# col.: the number of generated configurations for each model 


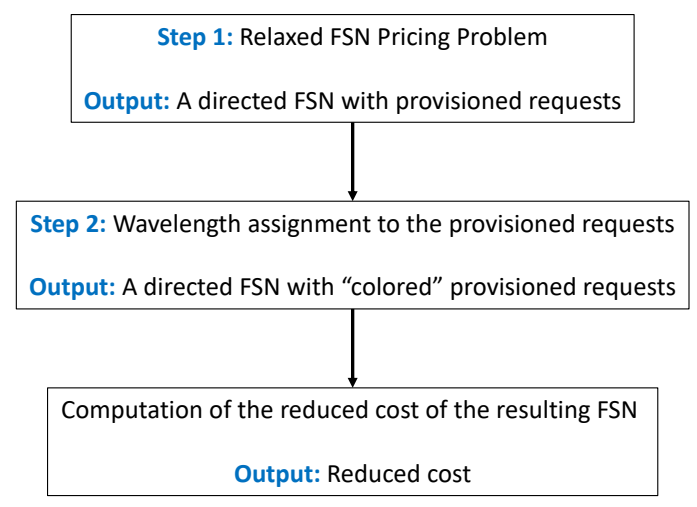

(a) Two step solution of the FSN pricing problem

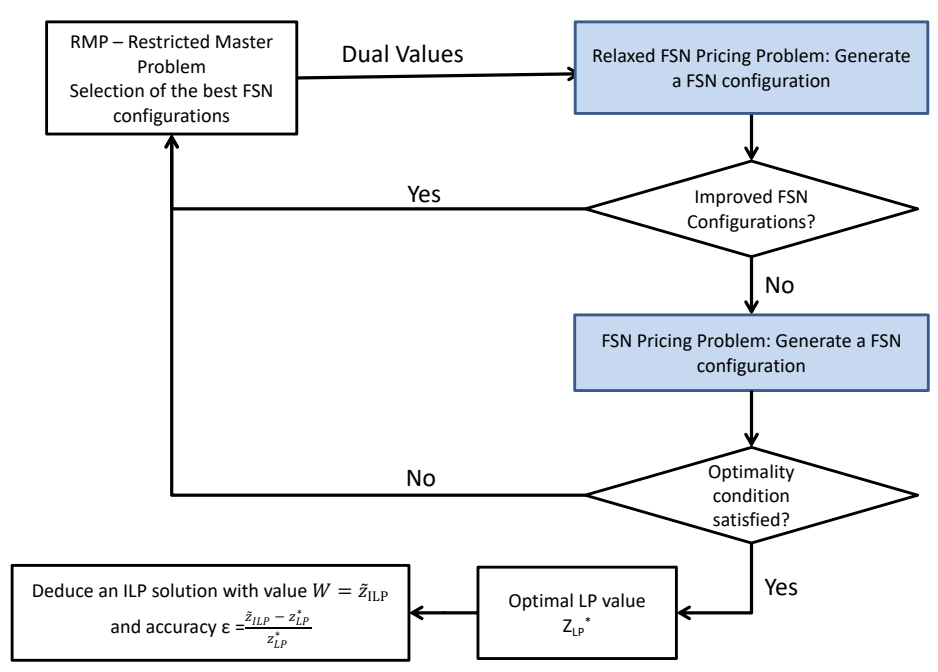

(b) Flowchart

Fig. 3: Flowcharts

TABLE II: Comparative results when using only one FSN

\begin{tabular}{|c|c|c|c|c|c|c|c|c|c|c|}
\hline & \multicolumn{4}{|c|}{ CG_FOP model (Jaumard et al. [11]) } & \multicolumn{4}{|c|}{ New DW_FOP model } & \multirow{2}{*}{$\begin{array}{l}\mathrm{CPU} \\
\text { ratio }\end{array}$} \\
\hline & & $\tilde{z}_{\mathrm{LP}}$ & \# col. & $W$ & $\mathrm{CPU}$ & $z_{\mathrm{LP}}^{\star}$ & \# col. & $W$ & $\mathrm{CPU}$ & \\
\hline \multirow{5}{*}{ Set 1} & \begin{tabular}{|l|l|} 
Italy \\
\end{tabular} & 41.0 & $180 / 516$ & 41 & $23 \mathrm{~h} 10 \mathrm{~m}$ & 40 & 1 & 40 & $10 \mathrm{~m} 06 \mathrm{~s}$ & $99.27 \%$ \\
\hline & California & 125.6 & $13 / 890$ & 126 & $1 \mathrm{~d} 6 \mathrm{~h} 46 \mathrm{~m} 47 \mathrm{~s}$ & 125 & 2 & 125 & $12 \mathrm{~m} 46 \mathrm{~s}$ & $99.31 \%$ \\
\hline & Germany & 120.5 & $7 / 813$ & 125 & $2 \mathrm{~d} 6 \mathrm{~h} 35 \mathrm{~m} 35 \mathrm{~s}$ & 123 & 2 & 123 & $50 \mathrm{~m} 30 \mathrm{~s}$ & $98.46 \%$ \\
\hline & Cost239 & 49.25 & $69 / 557$ & 51 & $1 \mathrm{~d} 9 \mathrm{~h} 35 \mathrm{~m} 20 \mathrm{~s}$ & 51 & 1 & 51 & $20 \mathrm{~m} 20 \mathrm{~s}$ & $98.99 \%$ \\
\hline & USA & 61.0 & $118 / 620$ & 61 & $6 \mathrm{~d} 11 \mathrm{~h} 45 \mathrm{~m} 43 \mathrm{~s}$ & 61 & 2 & 61 & $10 \mathrm{~m} 11 \mathrm{~s}$ & $99.89 \%$ \\
\hline & & & & & & & & & average & $99.18 \%$ \\
\hline \multirow{5}{*}{ Set 2} & Italy & & & & & 92 & 1 & 92 & $12 \mathrm{~m} 14 \mathrm{~s}$ & \\
\hline & California & & & & & 272 & 1 & 272 & $1 \mathrm{~h} 11 \mathrm{~m} 34 \mathrm{~s}$ & \\
\hline & Germany & & & & & 283 & 2 & 283 & $1 \mathrm{~h} 29 \mathrm{~m} 09 \mathrm{~s}$ & \\
\hline & Cost 239 & & & & & 112 & 2 & 112 & $34 \mathrm{~m} 25 \mathrm{~s}$ & \\
\hline & USA & & & & & 134 & 1 & 134 & $1 \mathrm{~h} 14 \mathrm{~m} 01 \mathrm{~s}$ & \\
\hline
\end{tabular}

- Model CG_FOP: the number of generated FSN configurations and the number of generated wavelength configurations, i.e., $180 \mathrm{FSN}$ configurations and 516 wavelength configurations for the Italy data instance in Table II.

- Model DW_FOP: the number of generated FSN configurations

- $W$ : an upper bound on the minimum number of required wavelengths in order to provision all requests

- CPU: computational times for reaching the $\varepsilon$-optimal solution associated with the $W$ value.

1) Single FSN case: For the single FSN case, we did not recourse to an initial solution, and solution of both models are done starting with an empty initial set of variables/columns.

The first striking result is the reduction of the computation time of $99 \%$ on average. We observe that all instances are solved exactly with the new DW_FOP model as the lower and upper bounds are equal. This was not the case with the CG_FOP model for which the accuracy of the solutions was ranging between $0 \%$ and $3.7 \%$. While the number of selected configurations is one in the ILP solution, we observe that the number of generated columns is pretty large for the previous CG_FOP model, which is likely to be the key explanatory factor for the huge differences for the computational results.

Results with the second set of requests also have the property that $W=z_{\mathrm{LP}}^{\star}$. However, computational times are increasing significantly, especially for the last network (USA).

2) Two FSN case: In the case of two FSNs, we use an initial solution made of a single FSN. We generate a single FSN to satisfy all the requests with the objective to minimize the link usage. In addition to the comparison with the CG_FOP model [11], we report some additional computational results of the literature, i.e., the numerical results of Tremblay et al. [8]. As for the single FSN case, we observe that the computation time needed for solving the new DW_FOP model is significantly smaller than for the CG_FOP model (average speedup of 55\%). Moreover, the solutions obtained with the DW_FOP model use less wavelengths than the solutions of both [11] and [8]. 
TABLE III: Comparative results with two FSNs

\begin{tabular}{|c|c|c|c|c|c|c|c|c|c|c|}
\hline & \multicolumn{10}{|c|}{ Provisioned filterless sub-networks on two trees } \\
\hline & \multirow{2}{*}{$\begin{array}{c}\text { Tremblay et al. }[8] \\
W\end{array}$} & \multicolumn{4}{|c|}{ CG_FOP model (Jaumard et al. [11]) } & \multicolumn{4}{|c|}{ DW_FOP model } & \multirow{2}{*}{$\begin{array}{l}\mathrm{CPU} \\
\text { ratio }\end{array}$} \\
\hline & & $z_{\mathrm{LP}}^{\star}$ & \# col. & $W$ & CPU & $\overline{z_{\mathrm{LP}}^{\star}}$ & \# col. & $W$ & CPU & \\
\hline Italy & 25 & 19.98 & $115 / 653$ & 23 & $58 \mathrm{~m} 57 \mathrm{~s}$ & 20 & 340 & 21 & $54 \mathrm{~m} 26 \mathrm{~s}$ & $7.66 \%$ \\
\hline California & - & 117.4 & $23 / 952$ & 122 & $1 \mathrm{~d} 15 \mathrm{~h} 37 \mathrm{~m}$ & 62.5 & 155 & 122 & $20 \mathrm{~h} 18 \mathrm{~m} 22 \mathrm{~s}$ & $48.74 \%$ \\
\hline Germ & 88 & 62.3 & 29/1610 & 73 & $2 \mathrm{~d} 22 \mathrm{~h} 41 \mathrm{~m} 31 \mathrm{~s}$ & 61.5 & 106 & 73 & $1 \mathrm{~d} 8 \mathrm{~h} 53 \mathrm{~m} 44 \mathrm{~s}$ & $53.47 \%$ \\
\hline Cost239 & - & 22.74 & $347 / 1183$ & 28 & $4 \mathrm{~d} 22 \mathrm{~h} 17 \mathrm{~m} 37 \mathrm{~s}$ & 23 & 270 & 25 & $1 \mathrm{~d} 0 \mathrm{~h} 47 \mathrm{~m} 07 \mathrm{~s}$ & $79.05 \%$ \\
\hline USA & - & 41.24 & $117 / 1011$ & 53 & $11 \mathrm{~h} 51 \mathrm{~m} 43 \mathrm{~s}$ & 31.25 & 302 & 53 & $1 \mathrm{~h} 40 \mathrm{~m} 05 \mathrm{~s}$ & $85.94 \%$ \\
\hline & & & & & & & & & average & $54.97 \%$ \\
\hline
\end{tabular}

TABLE IV: Network parameters for filterless solutions with three trees

\begin{tabular}{|c|c|c|c|c|c|c|c|c|c|c|}
\hline & \multicolumn{10}{|c|}{ Provisioned filterless sub-networks on three trees } \\
\hline & \multirow{2}{*}{$\frac{\text { Result of Tremblay } \text { et al. }[8]}{W}$} & \multicolumn{4}{|c|}{ CG FOP model (Jaumard et al. [11]) Result } & \multicolumn{4}{|c|}{ DW_FOP model Result } & \multirow{2}{*}{$\begin{array}{l}\mathrm{CPU} \\
\text { ratio }\end{array}$} \\
\hline & & $\tilde{z}_{\mathrm{LP}}$ & \# col. & $W$ & $\mathrm{CPU}$ & $\overline{z_{\mathrm{LP}}^{\star}}$ & \# col. & $W$ & CPU & \\
\hline California & 120 & 113.4 & $115 / 1040$ & 120 & $5 \mathrm{~d} 10 \mathrm{~h} 27 \mathrm{~m} 29 \mathrm{~s}$ & 41.67 & 85 & 120 & $19 \mathrm{~h} 24 \mathrm{~m} 37 \mathrm{~s}$ & $85.12 \%$ \\
\hline Cost 239 & - & 15.7 & $357 / 1386$ & 25 & $3 \mathrm{~d} 19 \mathrm{~h} 9 \mathrm{~m} 34 \mathrm{~s}$ & 15.33 & 201 & 17 & $39 \mathrm{~m} 44 \mathrm{~s}$ & $99.27 \%$ \\
\hline & & & & & & & & & average & $92.195 \%$ \\
\hline
\end{tabular}

Another interesting observation is the reduction of the number of wavelengths when moving from a single FSN to two FSNs. More precisely, we reduce on average the number of wavelengths by 21 , and the improvements range from 3 to 50 depending on the instance.

3) Three FSN case: In this experiment, we have changed Constraint (5) in order to select exactly $n_{\mathcal{F}}=3$ FSNs instead of at most $n_{\mathcal{F}}$ FSNs. With this modification, we observe that some instances are no longer feasible, i.e., for some instances it is not possible to partition the network and the set of requests into three FSNs while a solution with two FSNs is possible. Consequently, we analyse only the two instances for which a solution using three FSNs exists, namely California and Cost239.

As for the other experiments, we observe a significant reduction of the resolution time compared to the CG_FOP model (92\% faster on average). Furthermore, we obtain a solution for the Cost239 instance using only 17 wavelengths instead of 25 , which is a significant improvement.

\section{Impact of the number of FSNs}

In this last experiment, we compare the solutions for the USA network, when moving from one to two FSNs.

Figure 4(a) represent the physical fiber connectivity of USA network. Figure 4(b) depicts the single spanning tree solution of USA network, and Figure 4(c) describes the solution with two FSNs.

In both Figure 4(b) and 4(c), on each link that is used in the network provisioning, we indicate different numbers. Bidirected links indicate that the edge is used is both directions, while uni-directed links mean the edge is used in only one direction. For each direction, the numbers define the number of used wavelengths on the plain links, while they indicate the number of wasted wavelengths (unfiltered channels) on the dash links.

We observe that even in the case of two FSNs, one of them has a spanning tree as a backbone tree.

\section{Conclusions}

In this paper, we proposed a new mathematical model, DW_FOP, for the design of filterless optical networks that significantly improves upon the mathematical model and algorithm proposed in [11], thanks to a decomposition into a single pricing problem combining the definition of a filterless optical sub-network and its full provisioning, including the wavelength assignment. The DW_FOP model is much faster to solve and provides better solutions than previous proposals.

In the future, we plan to enhance further the model in order to handle multi-unit requests, without breaking them into single unit requests.

\section{REFERENCES}

[1] Cisco Annual Internet Report (2018-2023), CISCO, 2020.

[2] P. Pavon-Marino, F.-J. Moreno-Muro, M. Garrich, M. Quagliotti, E. Riccardi, A. Rafel, and A. Lord, "Techno-economic impact of filterless data plane and agile control plane in the 5G optical metro," Journal of Lightwave Technology, vol. 38, no. 15, pp. $3801-3814,2020$.

[3] J. M. Simmons, Optical Network Design and Planning, 2nd ed. Springer, 2014.

[4] B. Shariati, M. Ruiz, F. Fresi, A. Sgambelluri, F. Cugini, and L. Velasco, "Real-time optical spectrum monitoring in filterless optical metro networks," Photonic Network Communications, vol. 40, no. 1, pp. 1 13, 2020.

[5] A. Tzanakaki and M. O'Mahony, "Analysis of filterless wavelength converters employing cross-gain modulation in semiconductor optical amplifiers," in Conference on Lasers and Electro-Optics (CLEO), Baltimore, MD, USA, 1999, pp. 433 - 434.

[6] J.-P. Savoie, C. Tremblay, D. Plant, and M. Bélanger, "Physical layer validation of filterless optical networks," in European Conference on Optical Communication (ECOC), Torino, Italy, Sept. 2010, pp. $1-3$.

[7] A. Dochhan, R. Emmerich, P. W. Berenguer, C. Schubert, J. Fischer, M. Eiselt, and J.-P. Elbers, "Flexible metro network architecture based on wavelength blockers and coherent transmission," in 45th European Conference on Optical Communication (ECOC), Dublin, Ireland, 2019, pp. $1-4$.

[8] C. Tremblay, E. Archambault, M. Bélanger, J.-P. Savoie, F. Gagnon, and D. Plant, "Passive filterless core networks based on advanced modulation and electrical compensation technologies," Telecommunication Systems, vol. 52, no. 4, pp. $167-181$, January 2013.

[9] O. Ayoub, S. Shehata, F. Musumeci, and M. Tornatore, "Filterless and semi-filterless solutions in a metro-HAUL network architecture," International Conference on Transparent Optical Networks (ICTON), pp. $1-4,2018$. 


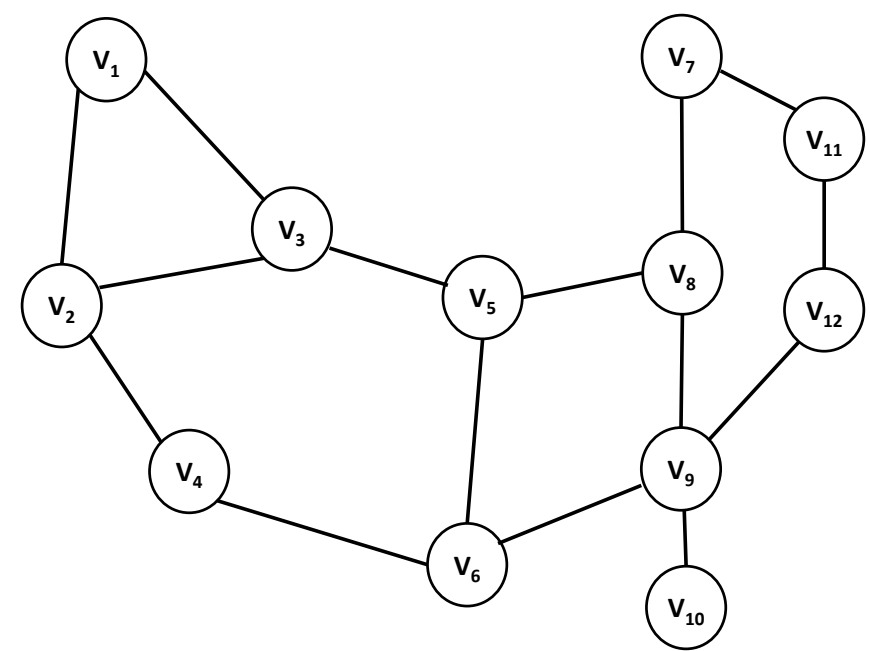

(a) Physical network

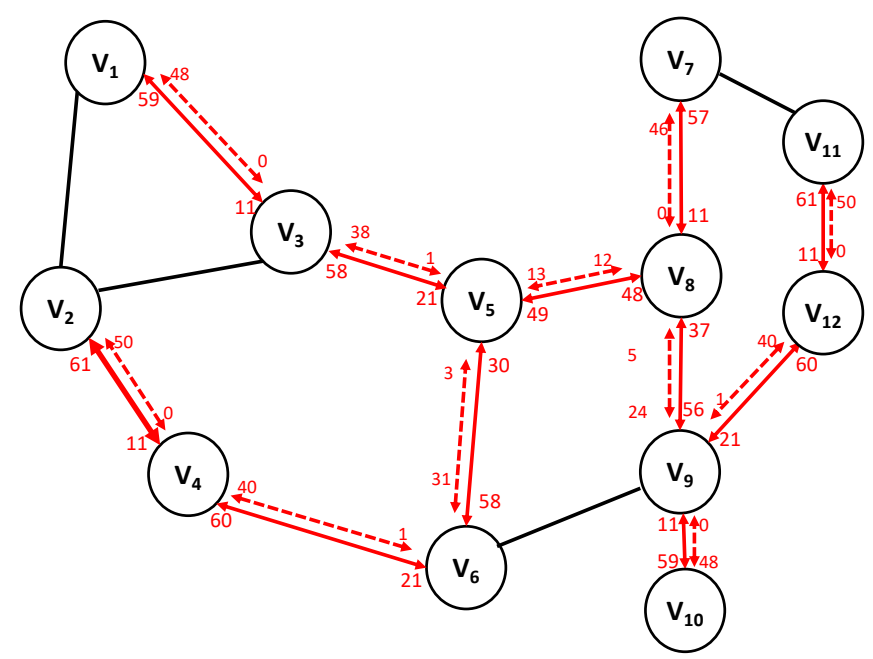

(b) Single spanning FSN provisioning

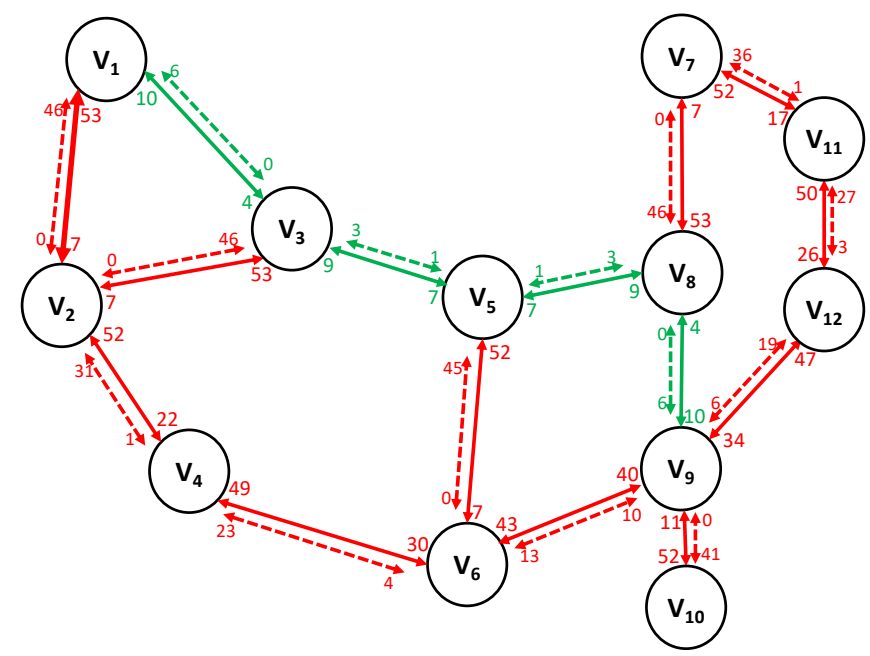

(c) Two FSN fiber trees provisioning
[10] B. Jaumard, Y. Wang, and N. Huin, "Optimal design of filterless optical networks," International Conference on Transparent Optical Networks (ICTON), pp. 1-5, 2018.

[11] B. Jaumard and Y. Wang, "A two sub-problem decomposition for the optimal design of filterless optical networks," 2021, arXiv:2101.01022.

[12] O. Ayoub, A. Bovio, F. Musumeci, and M. Tornatore, "Survivable virtual network mapping in filterless optical networks," in International Conference on Optical Network Design and Modeling (ONDM), Castelldefels, Barcelona, Spain, 2020, pp. $1-6$.

[13] D. Uzunidis, M. Presi, A. S. aand F. Paolucci, A. Stavdas, and F. Cugini, "Bidirectional single-fiber filterless optical networks: modeling and experimental assessment," Journal of Optical Communications and Networking, vol. 13, no. 6, pp. C1 - C9, January 2021.

[14] O. Ayoub, F. Fatima, A. Bovio, F. Musumeci, and M. Tornatore, "Trafficadaptive re-configuration of programmable filterless optical networks," IEEE International Conference on Communications - ICC, pp. $1-6$, 2020.

[15] V. Chvatal, Linear Programming. Freeman, 1983.

[16] G. Nemhauser and L. Wolsey, Integer and Combinatorial Optimization. Wiley, 1999, reprint of the 1988 edition.

[17] M. Aguayo, S. C. Sarin, and H. D. Sherali, "Solving the single and multiple asymmetric traveling salesmen problems by generating subtour elimination constraints from integer solutions," IISE Transactions, vol. 50, no. 1, pp. $45-53,2018$.

[18] A. Mehrotra and M. Trick, "A column generation approach for graph coloring," INFORMS Journal on Computing, vol. 8, no. 4, pp. 344-354, 1996.

[19] S. Orlowski, R. Wessäly, M. Pióro, and A. Tomaszewski, "SNDlib 1.0 -survivable network design library," Networks, vol. 55, no. 3, pp. 276-286, 2010

Fig. 4: Solution of US 12 nodes network 